\title{
THE STANMORE HINGED KNEE ARTHROPLASTY
}

\author{
A. W. F. LETTIN, L. J. DELISS, J. S. BLACKBURNE, J. T. SCALES
}

From the Institute of Orthopaedics, and St Bartholomew's Hospital, London

\begin{abstract}
The Stanmore hinged total knee replacement was introduced in 1969 for severe destructive arthropathy of the knee, and the results of one hundred consecutive operations are presented after an average interval of two and a half years. Insertion of the prosthesis relieved pain in 94 per cent, improved the range of movement in 67 per cent, invariably restored stability and corrected valgus or varus deformity. Gross flexion contractures were improved but not always fully corrected. Serious complications were few, though of three cases of deep infection two came to amputation and one to fibrous ankylosis. There were no mechanical failures of components of the prosthesis.
\end{abstract}

The Stanmore hinged knee prosthesis was developed in 1968 from a hinged knee replacement made substantially of acrylic polymer with a nylon axle, which had been produced in 1952 to replace the lower end of a femur affected by hydatid disease (Burrows

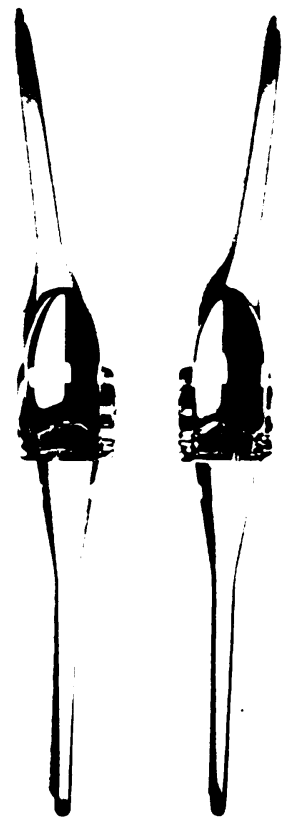

Fig. 1

The Mark $4 b$ replacement.

This design has not been changed since 1972 .

1968). Two years later a metallic prosthesis was made to replace the lower femur and knee in a patient with a recurrent giant-cell tumour, and five prostheses of similar design were subsequently used with encouraging results (Burrows, Wilson and Scales 1975).
The early designs of the prosthesis were made either of Titanium 160 with Vinertia bearings or entirely of cobalt-chromium-molybdenum alloy. Twenty such prostheses were used and that experience led to the present design (Fig. 1) which has not been changed since 1972 (Wilson, Lettin and Scales 1974). It is made of cobalt-chromium-molybdenum alloy with bushes of ultra-high molecular weight polyethylene and thrust washers in which a stationary metal axle is retained by a circlip of Titanium 318. The design features long oval tapering medullary stems anchored to the bone by Surgical Simplex cement, and a large articular surface with which the patella is in contact throughout the range of movement. There are mirror-image prostheses for right and left knees.

\section{SELECTION OF PATIENTS}

The aim of prosthetic replacement of the knee is to relieve pain, to correct deformity, and to restore a functional range of movement with stability. It has been reserved for those patients judged unsuitable for lesser procedures but no patient has been rejected on account of gross deformity, instability or limitation of movement.

The first 100 Stanmore prostheses were inserted in seventy-five patients between March 1969 and December 1976 by one of the authors (AWFL) or his assistants. Four patients died before the review from unrelated causes; the other seventy-one (ninety-six knees) have been examined personally (by JSB or LJD). The first twenty prostheses, the results of sixteen of which have been reviewed, were the earlier design and the last eighty were the current model (Fig. 1).

Twenty-five patients (thirty-one knees) suffered from osteoarthritis and forty-six (sixty-five knees) from

A. W. F. Lettin, M.S., F.R.C.S., Consultant Orthopaedic Surgeon, St Bartholomew's Hospital, London EC1A 7BE, England. L. J. Deliss, F.R.C.S., Senior Registrar, Royal National Orthopaedic Hospital, 234 Great Portland Street, London W1N 6AD, England. J. S. Blackburne, F.R.C.S., Consultant Orthopaedic Surgeon, Barnet General Hospital, Barnet, Hertfordshire EN5 3DJ, England. Professor J. T. Scales, F.R.C.S., C.I.Mech.E., Institute of Orthopaedics, Brockley Hill, Stanmore, Middlesex HA7 4LP, England. Requests for reprints should be sent to Mr A. W. F. Lettin. 
rheumatoid arthritis, for which sixteen patients (twenty-four knees) were taking systemic steroids. There were twelve men and fifty-nine women, aged between forty-eight and eighty-seven years. Twenty-six patients had had previous operations on the affected knee (Table I) and sixty-six had significant symptoms from other major joints. Two-thirds of the patients had had symptoms arising from the knee for ten years or more.

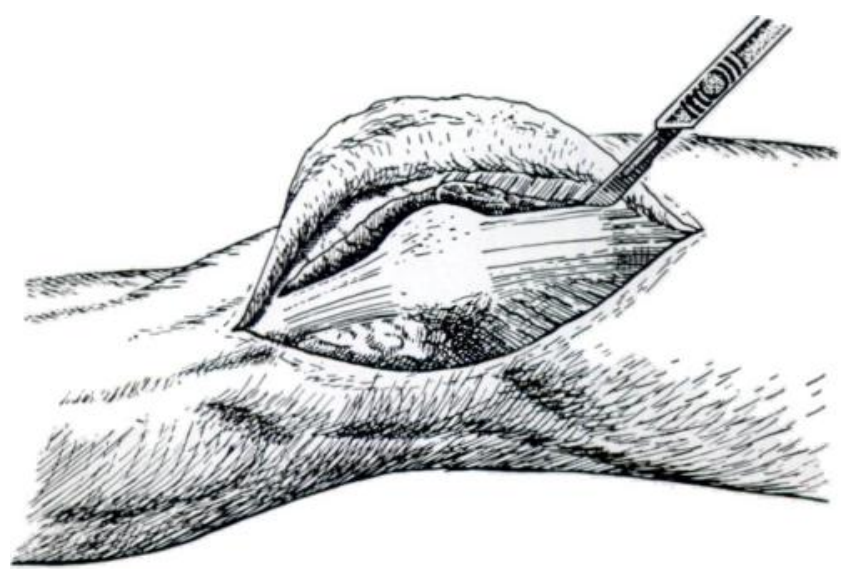

Fig. 2

A diagram of the approach to a left knee joint.

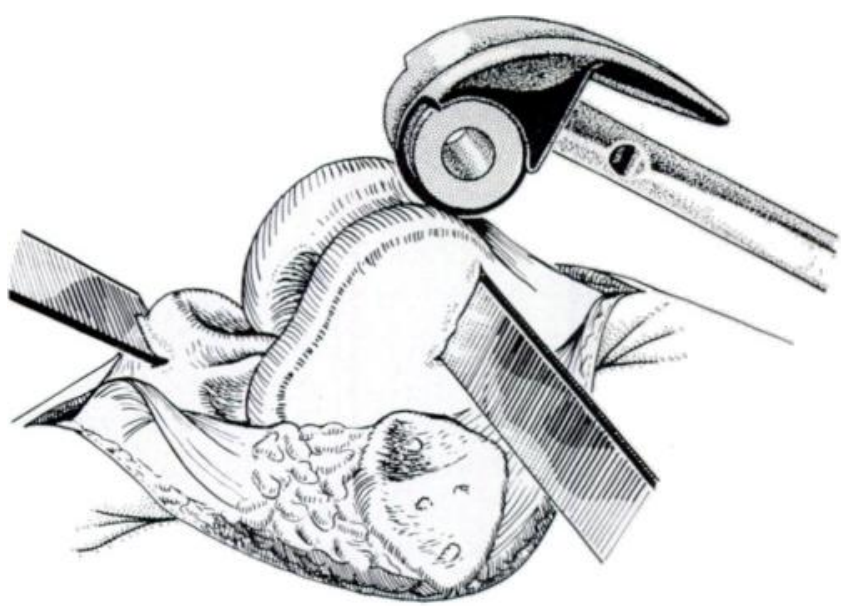

Fig. 5

A drawing to show the technique of resection.

\section{OPERATIVE TECHNIQUE}

A high tourniquet is used and a lateral parapatellar incision made. The flap is reflected medially and the joint is opened through a medial parapatellar incision in the capsule extending upwards from the tibial tubercle as far as is necessary to allow the patella to be dislocated laterally (Fig. 2). The knee is then flexed, exposing the femoral condyles from which the soft tissues are dissected. The femoral condyles are resected at right angles anteroposteriorly (Fig. 3) and in anatomical valgus of about 10 degrees (Fig. 4). The plane of section is determined by placing the femoral component parallel to the femur with its distal surface in the same plane as that of the condyles, which are marked at the level of the plateau on the prosthesis (Fig. 5). The posterior parts of the femoral condyles are trimmed flush with the posterior cortex (Fig. 3) in order to allow full flexion of the artificial joint.

The tibia is resected just above the tubercle at right angles to the shaft in both planes (Figs. 3 and 4). In preparing the medullary cavities only sufficient cancellous bone is curetted to take the stems of the prosthesis, because fixation of the prosthesis depends on anchoring the long stems in the medullary canals of the tibia and femur rather than in the soft cancellous bone of the condyles. The congruity between patella and prosthesis is checked with a template (Fig. 6) and any
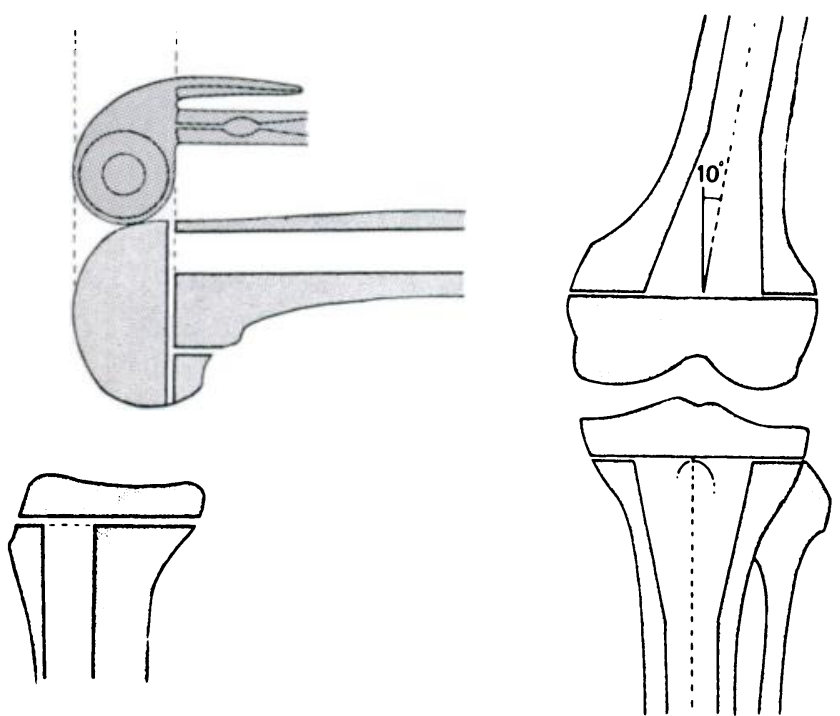

Fig. 3

Fig. 4

Figures 3 and 4-Line diagrams of the resection of a left knee and curettage of the medullary cavities in the lateral plane (Fig. 3) and in the frontal plane (Fig. 4).

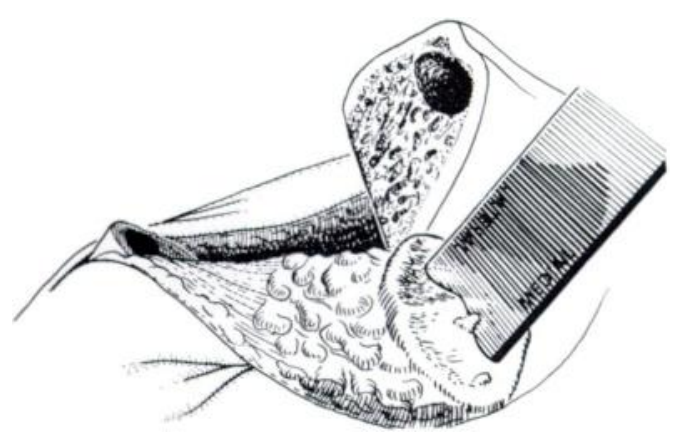

Fig. 6

The articular surface of the patella is checked with the template to ensure that it will articulate correctly with the patella-bearing surface of the femoral component.

peripheral osteophytes causing incongruity are nibbled away.

Both components are then temporarily "tried" to ensure that a minimum of 90 degrees of flexion can take place. The stability of the patella and the alignment of the extensor mechanism are also checked.

The tibial and femoral components are simultaneously cemented in position and again articulated with the trial axle. Any excess of cement is removed and the knee held extended and still until the remainder has cured. Irrigation with saline solution removes any loose particles of cement or bone. The trial axle is replaced by the permanent axle, which is held firmly in place by a circlip. The joint is irrigated once more and five grams of chloramphenicol are instilled before closure 
with suction drainage. After the application of a firm bandage over cotton-wool the tourniquet is released and the knee splinted with a back-slab.

Isometric quadriceps exercises are started as soon as possible. The wound is redressed when the deep and superficial drains are removed after forty-eight hours. Weight-bearing in the back-slab may be started as soon as the general condition allows, and the back-slab is discarded once quadriceps control has been regained. Flexion is allowed as soon as the condition of the wound permits, usually between the seventh and tenth day. A gentle manipulation under anaesthetic is occasionally needed to improve the range of flexion.

Table I. Previous treatment

\begin{tabular}{|ll|}
\hline One operation & \\
Double osteotomy & 9 \\
Tibial osteotomy & 3 \\
Synovectomy & 5 \\
Patellectomy & 4 \\
Walldius prosthesis & 1 \\
Macintosh prostheses & 1 \\
Two operations & \\
Synovectomy + tibial osteotomy & 2 \\
Patellectomy + double osteotomy & 1 \\
\hline
\end{tabular}

\section{COMPLICATIONS}

The following complications occurred in the series of 100 consecutive operations.

Delayed healing. This occurred in twenty-nine knees, sixteen of which were affected by rheumatoid arthritis, and six in patients receiving systemic steroid therapy. In most cases the delay was associated with a serosanguinous or purulent discharge, from ten of which organisms were cultured. An area of skin necrosis developed in nine knees, four of which had scars from previous operations; in three of these four the necrotic area was excised and covered by a rotation flap. In the other six the slough separated and the wound finally healed. The lack of more serious complications in these circumstances is attributed to the separation of the skin and capsular incisions (Fig. 2) which helped to ensure that the prosthesis was covered by healthy tissue even in the presence of a superficial complication. Two patients, however, did develop a persistent shallow sinus; one closed spontaneously and the other healed after rotation of a local flap.

Deep infection. This occurred on three occasions.

A woman aged seventy-six with rheumatoid arthritis, who had had a double osteotomy on the same knee and a total replacement of the opposite knee developed a deep infection one month after primary healing of the wound. This was treated successfully by oral antibiotics and closed continuous irrigation with diethanolamine fucidate for ten days. Twelve months later she had $\mathbf{8 0}$ degrees of painless movement.

A man of fifty-four, crippled by long-standing generalised rheumatoid disease developed flexion contracture of $\mathbf{4 0}$ degrees after a MacIntosh arthroplasty and was confined to a wheelchair. A Stanmore prosthesis was inserted and the flexion contracture was corrected. Unfortunately a large haematoma formed as a result of failure of the suction drainage, and although this was evacuated forty-eight hours after operation, the skin sloughed and required a local rotation flap. Four months later the patient was readmitted with a discharging sinus. Despite further plastic procedures and continuous irrigation the prosthesis became exposed and an above-knee amputation was performed at the patient's request. The patient is now able to walk with an artificial limb and crutches.

Above-knee amputation was also carried out on a woman of sixty-two three years after replacement. She had previously had a patellectomy through a transverse incision, and soon after the arthroplasty developed inflammation of the wound which resolved with antibiotic therapy. Two years later the transverse scar broke down, exposing the prosthesis. A rotation flap was initially successful but the wound again broke down. Skin tests showed sensitivity to cobalt and nickel.

Loosening. Five prostheses have become loose.

One case was associated with a fracture of the lateral femoral condyle and another with a spiral fracture of the femoral shaft, both resulting from falls. A third prosthesis became loose within three months of operation, apparently due to insufficient cement around the femoral component. All three were successfully replaced. A fourth prosthesis is known to be loose but the patient considers that the symptoms do not warrant treatment.

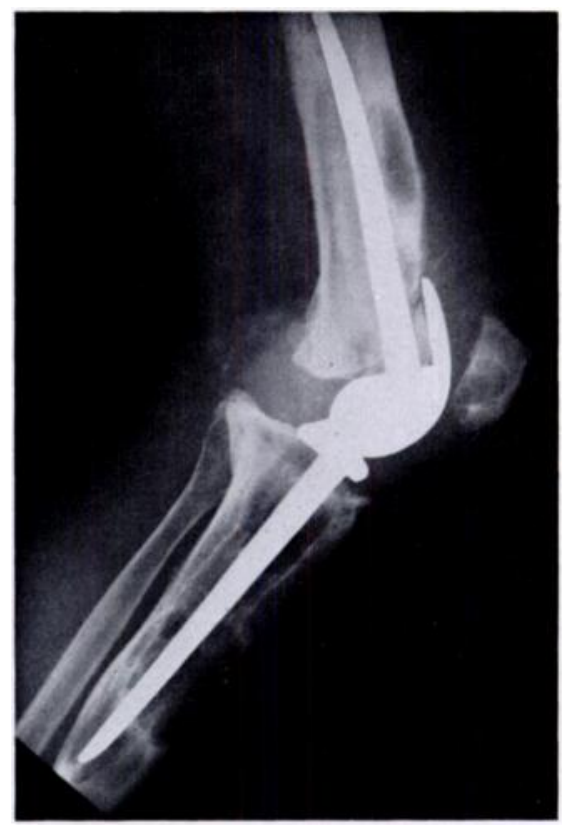

Fig. 7

A radiograph showing multiple lytic areas around the prosthesis.

The fifth patient, a man of sixty-eight with bilateral osteoarthritis of the knees, complained of some discomfort in the knee from the time of operation. However, he had 90 degrees of flexion and was not incapacitated. Six months later he complained of pain in the shin and local tenderness, and the radiograph showed a periosteal reaction. The symptoms subsided after a short course of antibiotics but he returned a year later with severe pain. Radiographs now revealed multiple lytic areas around the prosthesis in the cortex of the femur and the tibia, and also in the patella (Fig. 7). The prosthesis and cement, which were 
presumed to be infected, were removed and compression arthrodesis was attempted, but there was no evidence of infection at operation and no organisms were cultured. The wound healed normally but despite prolonged splintage he was left with a fibrous ankylosis.

It is perhaps worthy of note that two patients sustained a fracture of the femoral neck and one of the femoral shaft on the same side as the artificial joint without loosening of the prosthesis. All three were treated conventionally.

Disruption of the extensor mechanism. In two patients the patellar ligament was avulsed at the time of operation but was successfully reattached. Two patients later avulsed the patellar ligament from the tibial tubercle; one has no disability and can walk using a stick, and the other walks with a polythene support. In five knees (three patients), all with the early type of prosthesis, the patella dislocated, producing a gross extension lag. In one patient the patella was removed without improvement and in another the patellar ligament has been successfully realigned.

Other complications. Occasionally a symptomless lateral subluxation of the patella has been noted, particularly in a knee originally with a valgus deformity. One patient sustained an undisplaced longitudinal fracture of the femoral shaft during the operation. This was treated by immediate cerclage wiring and the progress after operation was uneventful. Two patients with fixed flexion deformities of 40 degrees developed a lateral popliteal nerve palsy after knee replacement; both recovered within six months.

\section{RESULTS}

Because the results of the early design, apart from the higher incidence of patellofemoral problems, differ little from those with the later design, they are not separated in the final assessment and are set out in Tables II, III and IV.

Patient's assessment. This was regarded as the most important measure of success of the procedure. Most of the patients have been pleased with the replacement.

Pain. Before operation the pain was either constant or severe enough to interrupt sleep, except for three ankylosed knees. After replacement no patient had pain more severe than before; in fact ninety knees ( 94 per cent) were entirely pain-free or gave rise to only occasional discomfort considered insignificant by the patient. This discomfort was patellofemoral in nine knees; six of those prostheses were of the early design without an articulating surface for the patella.

Deformity. Before replacement half of the knees had a flexion contracture of more than 10 degrees; this proportion was reduced to 9 per cent. All ten knees with a flexion contracture greater than 30 degrees were improved, but even so a flexion contracture exceeding 30 degrees remained in three.

The most severe flexion contractures occurred in a man of sixty-six with generalised rheumatoid disease who had been confined to a wheelchair for six years; both knees were ankylosed at a right angle (Figs. 8 and 9). Although complete correction was achieved by soft tissue release and bone resection, the patient was left with marked extension lag which eventually led to flexion contracture of 40 degrees on one side and of 30 degrees on the other (Fig. 10). Even so the knees gained 50 and 60 degrees of movement and the patient is able to walk with crutches. He considers the ability to "lie straight in bed at night" a major benefit.

Instability. A constrained prosthesis with a built-in tibiofemoral "standing angle" should inevitably correct

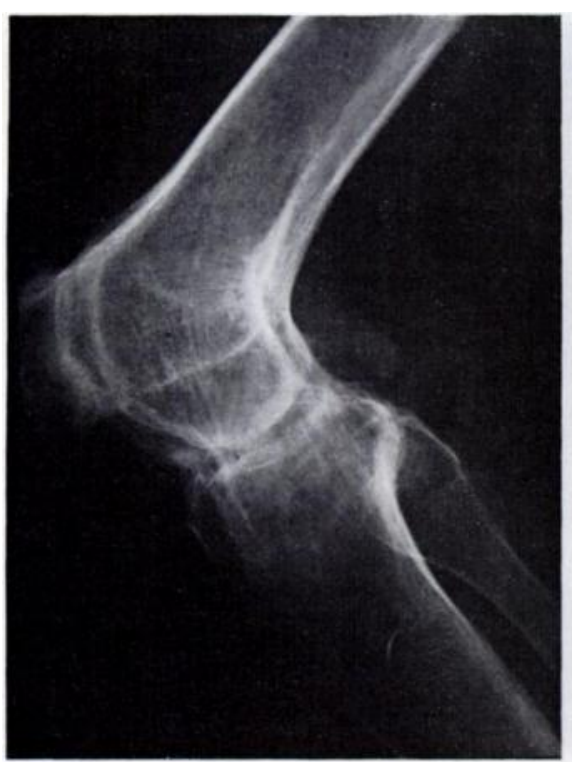

Fig. 8

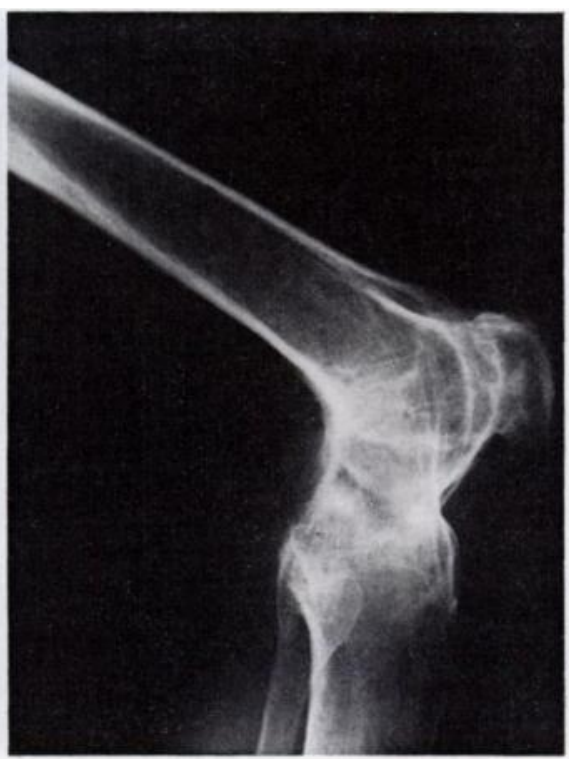

Fig. 9

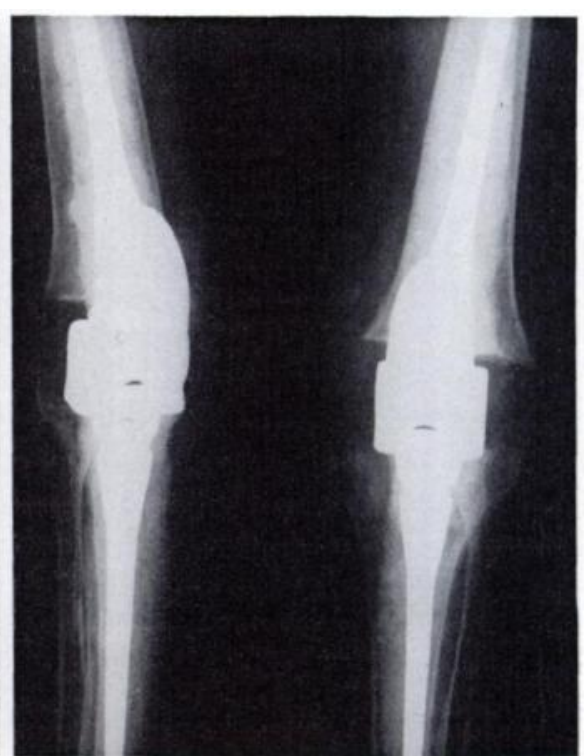

Fig. 10

Figures 8 and 9-Right and left knees ankylosed at 90 degrees before operation. Figure 10-Successful replacement of both knees. 
Table II. Clinical assessment before operation

\begin{tabular}{|c|c|c|c|c|c|c|c|c|c|c|c|}
\hline \multirow[b]{2}{*}{ Clinical features } & \multicolumn{2}{|c|}{ O } & \multicolumn{2}{|c|}{1} & \multicolumn{2}{|c|}{$\begin{array}{l}\text { Grade of assessment } \\
\mathbf{2}\end{array}$} & \multicolumn{2}{|c|}{3} & \multicolumn{2}{|c|}{4} & \multirow[b]{2}{*}{ Total } \\
\hline & Number & Per cent & Number & Per cent & Number & Per cent & Number & Per cent & Number & Per cent & \\
\hline Pain & - & - & 47 & 49 & 29 & 30 & 17 & 18 & 3 & 3 & 96 \\
\hline Ability to walk & & & & & & & & & & & \\
\hline Walking aid & \multicolumn{11}{|c|}{ Data not available } \\
\hline \multicolumn{12}{|l|}{ Gait } \\
\hline Flexion deformity & - & - & 10 & 10 & 16 & 17 & 22 & 23 & 48 & 50 & 96 \\
\hline Maximal flexion & - & - & $25^{*}$ & 26 & 9 & 9 & 42 & 44 & 20 & 21 & 96 \\
\hline Extension lag & - & - & 0 & 0 & 3 & 3 & 93 & 97 & 0 & 0 & 96 \\
\hline Valgus angle & - & - & 3 & 3 & 10 & 10 & 12 & 13 & 71 & 74 & 96 \\
\hline Varus angle & - & - & 0 & 0 & 1 & 1 & 7 & 7 & 88 & 92 & 96 \\
\hline Get out of chair & \multirow{2}{*}{\multicolumn{11}{|c|}{ Data not available }} \\
\hline Climb stairs & & & & & & & & & & & \\
\hline
\end{tabular}

* Five knees were ankylosed

Table III. Assessment by the patient after operation

\begin{tabular}{|c|c|c|c|c|c|c|c|c|c|c|c|}
\hline & \multicolumn{4}{|c|}{0} & \multicolumn{2}{|c|}{$\begin{array}{c}\text { Grade of assessmen } \\
\mathbf{2}\end{array}$} & \multicolumn{2}{|c|}{3} & \multicolumn{2}{|c|}{4} & \multirow[b]{2}{*}{ Total } \\
\hline & Number & Per cent & Number & Per cent & Number & Per cent & Number & Per cent & Number & Per cent & \\
\hline A & - & - & 8 & 8 & 3 & 3 & 32 & 34 & 53 & 55 & 96 \\
\hline B & \multicolumn{10}{|c|}{ Data not available } & \\
\hline
\end{tabular}

See text: "Functional Assessment", page 332.

Table IV. Clinical assessment after operation

\begin{tabular}{|c|c|c|c|c|c|c|c|c|c|c|c|}
\hline \multirow[b]{2}{*}{ Clinical features } & \multicolumn{2}{|c|}{ O } & \multicolumn{2}{|c|}{1} & \multicolumn{2}{|c|}{$\begin{array}{c}\text { Grade of assessment } \\
\qquad \mathbf{2}\end{array}$} & \multicolumn{2}{|c|}{3} & \multicolumn{2}{|c|}{4} & \multirow[b]{2}{*}{ Total } \\
\hline & Number & Per cent & Number & Per cent & Number & Per cent & Number & Per cent & Number & Per cent & \\
\hline Pain & - & - & 0 & 0 & 1 & 1 & 5 & 5 & 90 & 94 & 96 \\
\hline \multicolumn{12}{|l|}{ Ability to walk } \\
\hline Walking aid & \multicolumn{11}{|c|}{ Data not available } \\
\hline \multicolumn{12}{|l|}{ Gait } \\
\hline Flexion deformity & - & - & 3 & 3 & 2 & 2 & 4 & 4 & 84 & 91 & $93^{*+}$ \\
\hline Maximal flexion & - & - & 7 & 7 & 12 & 13 & 63 & 67 & 12 & 1.3 & $94^{*}$ \\
\hline Extension lag & - & - & 7 & 8 & 5 & 5 & 10 & 10 & 72 & 77 & 94 \\
\hline Valgus angle & - & - & 0 & 0 & 0 & 0 & 0 & 0 & 93 & 100 & 93 \\
\hline Varus angle & - & - & 0 & 0 & 0 & 0 & 0 & 0 & 93 & 100 & 93 \\
\hline Get out of chair & \multirow{2}{*}{\multicolumn{11}{|c|}{ Data not available }} \\
\hline Climb stairs & & & & & & & & & & & \\
\hline
\end{tabular}

*Two amputations. † One fibrous ankylosis.

For details of grades, see page 309. 


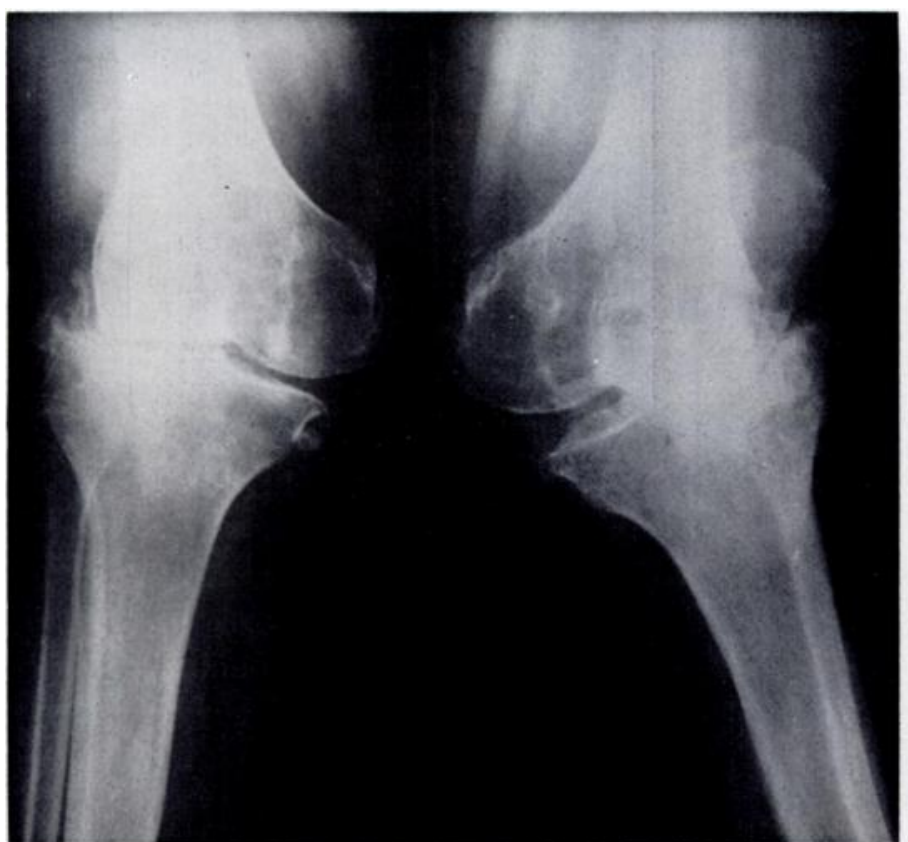

Fig. 11

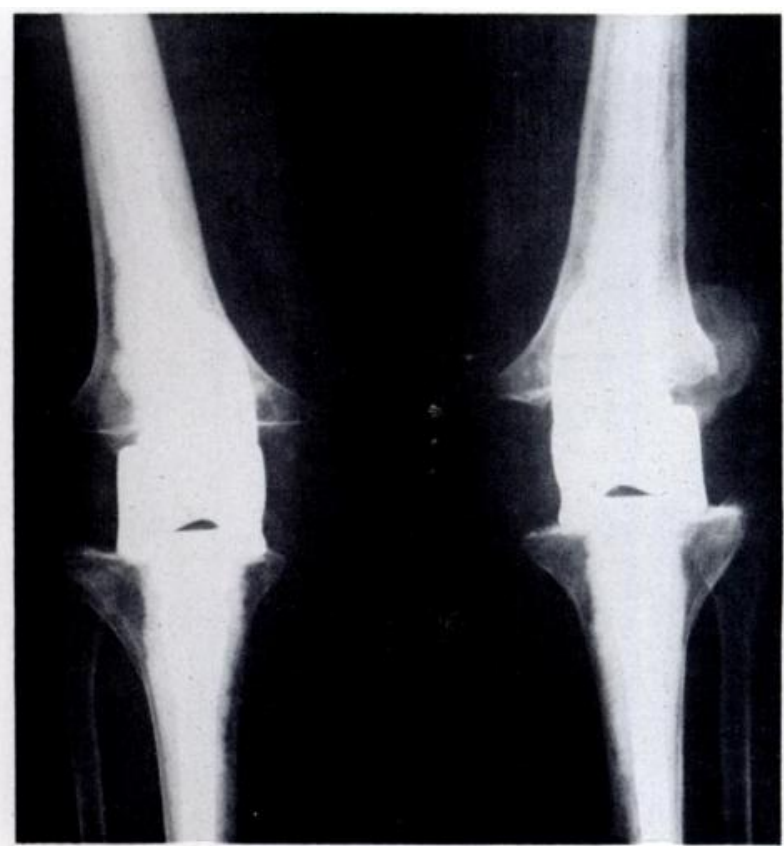

Fig. 12

Figures 11 and 12-Radiographs taken while weight-bearing before and after replacement, illustrating correction of valgus deformities and gross instability.

valgus or varus deformity and instability. An extension lag, however, may still occur. Seventy-two knees (77 per cent) had full active extension after replacement, but seven ( 8 per cent) had a lag in excess of 20 degrees from disruption of the extensor mechanism with surprisingly little untoward effect on function.

Two-thirds of the knees had considerable valgus or varus instability before operation but all the ninetythree joints with a prosthesis still in situ were clinically stable at review (Figs. 11 and 12). In spite of this some patients experienced a feeling of insecurity, particularly on going down stairs.

Movement. Many knees had retained a good range of movement before operation despite severe pain and deformity. Nevertheless the range of movement increased in sixty-four knees (67 per cent), the stiffest joints making the most notable gains.

Eighteen knees lost some of the range of movement, but with one exception they had 90 degrees or more of flexion before replacement and retained 85 degrees or more, the loss being of no functional significance.

Functional assessment. All but six patients said that replacement had improved their functional capacity.
Improvement, or the reverse, proved impossible to quantify because most of the patients had involvement of other joints which hindered walking or even rising from a chair. Objective functional improvement was frequently only apparent after further operations on other joints. The improved function often amounted to no more than the ability to perform a task more rapidly, but many patients were able to shop, dance or work in the garden for the first time for many years. In five patients the lack of functional improvement was associated with complications which required further surgical treatment.

\section{CONCLUSIONS}

The operative procedure described is straightforward and minor errors do not appear to jeopardise the outcome. Few special instruments are required. Up to the time of review there has been no mechanical failure or wear of the prostheses. The operation fulfils the aims of prosthetic replacement, consistently relieving pain, correcting deformity, restoring stability and either maintaining or improving movement, and may prove to be a satisfactory long-term treatment for patients suffering from painful destructive arthritis.

We would like to thank the Departments of Medical Illustration at the Royal National Orthopaedic Hospital and at St Bartholomew's Hospital for preparation of the illustrations.

\section{REFERENCES}

Burrows, H. J. (1968) Major prosthetic replacement of bones: lessons learnt in seventeen years. Journal of Bone and Joint Surgery, 50-B, 225-226.

Burrows, H. J., Wilson, J. N., and Scales, J. T. (1975) Excision of tumours of humerus and femur, with restoration by internal prostheses. Journal of Bone and Joint Surgery, 57-B, 148-159.

Walldius, B. (1957) Arthroplasty of the knee joint using an endoprosthesis. Acta Orthopaedica Scandinavica, Supplement 24.

Wilson, J. N., Lettin, A. W. F., and Scales, J. T. (1974) Twenty years of evolution of the Stanmore hinged total knee replacement. Proceedings of Conference on Total Knee Replacement, pp. 61-67. London: Institution of Mechanical Engineers. 